Original Article

\title{
EFFICACY OF FUNCTIONAL NEUROMUSCULAR ELECTRICAL STIMULATION (FNMES) IN THE IMPROVEMENT OF HAND FUNCTIONS IN ACUTE STROKE SURVIVALS
}

\author{
Mohamed Faisal C.K. ${ }^{1}$, Priyabandani Neha Om Prakash ${ }^{2}$, Ajith S. ${ }^{3}$ \\ ${ }^{1}$ Professor \& Vice Principal, ${ }^{2}$ Physiotherapist, ${ }^{3}$ Assistant Professor, \\ Nitte Institute of Physiotherapy, Nitte University, Mangalore, India - 575018
}

\author{
Correspondence:
}

Mohamed Faisal C.K.

Professor \& Vice Principal, Nitte Institute of Physiotherapy, Nitte University, Mangalore, India-575018. E-mail : faisal77mpt@gmail.com

\section{Abstract:}

Stroke is a worldwide health problem. Hand function is one of the important factors which are affected in stroke. Stroke patients are usually given a conventional physiotherapy but if an additional FNMES is given it might show better improvement. By keeping these facts in view, the present study aims at evaluating and comparing the efficacy of conventional physiotherapy and adding FNMES will make any better outcome in the acute stroke survivals. The subjects were randomly assigned to any of the two groups; control group consisted of 15 subjects who received only conventional therapy for 4 weeks and experimental group consisting of 15 subjects who received an additional FNMES along with conventional physiotherapy for 4 weeks. The hand function was assessed on day 1 and to know the recovery, at the end of four weeks of intervention with the help of action research arm test (ARAT) and box and block test (BBT). At the end of 4 weeks of intervention both the groups showed significant improvements. On ARAT, control group showed a mean of 10.2000 whereas, experimental group showed mean of 20.8000 with $p=0.001(p \leq 0.05)$ and on BBT, the control group showed a mean of 21.666 and experimental group showed 30.933 with $p=0.41$ ( $p \leq 0.05$ ). Therefore the study concludes that, though there was improvement in both the groups, the experimental group who received an additional FNMES along with conventional physiotherapy showed better improvement in hand functions in the acute stroke survivals.

Keywords : Stroke, Hand function, FNMES, ARAT, BBT.

\section{Introduction :}

Stroke is a clinical syndrome characterized by the sudden development of a persisting focal neurological deficit. Although the prevalence of stroke appears to be comparatively less in India than in developed countries, it is likely to increase proportionally with the increase in life expectancy. $^{1,2}$. The prevalence of stroke in India was estimated as 203 per 100,000 populations. The best estimate derived was 102,000 deaths; which represented $1.2 \%$ of total deaths in the country. ${ }^{3}$ The incidence of stroke

\begin{tabular}{|c|}
\hline Access this article online \\
\hline Quick Response Code \\
\hline \\
\end{tabular}
is about $19 \%$ higher in males than in females. Stroke is one of the major causes of human morbidity and mortality ${ }^{4}$.

Stroke or cerebrovascular accident is an acute onset of neurological dysfunction due to an abnormality in cerebral circulation with resultant signs and symptoms that correspond to the involvement of focal areas of brain. Hemiplegia may be the most obvious sign of cerebrovascular accident and a major concern for therapists ${ }^{5}$. Approximately half of all the stroke survivors are left with major problems in their extremity. The degree of motor recovery after stroke varies widely and is directly related to the degree of initial severity and interval from stroke to initiation of voluntary movement ${ }^{6}$. Both basic and clinical studies suggest that post stroke motor recovery or motor learning of paretic limb may be maximized by the active repetitive use of the affected limb. The stimulation from active rehabilitation and an enriched environment plays an important part in brain repair and recovery. ${ }^{7}$

Voluntary movement control is typically impaired after stroke. Movement control of the body on the contra lateral 
side of the brain lesion proceeds through stages of recovery in which, the sensory and motor functions are often reestablished abnormally. In the upper extremity after a period of flaccidity a common course of recovery includes the development of an uncontrolled flexion synergy. This pathological synergy is observed in the hemi paretic limb during efforts to use the arm for functional task. These disabilities lead to development of compensatory strategies for accomplishing daily needs and frequently minimize the use of the paretic arm and hand. ${ }^{8}$

Stroke is particularly prevalent in older people and the effects of stroke can be profound. Not only are the abilities to stand, balance and walk affected, but also the ability to use the upper limb and hand in its diversity of functions in everyday life is affected. Loss of independence of upper limb function contributes enormously to functional disability, affecting quality of life and independence in 'basic' (washing, grooming, feeding, dressing, etc.) and 'instrumental' activities (shopping, home/financial management, etc.) of daily living. , $^{9,10}$

The main indication for using the FNMES method is, a deficiency of muscle function, which may be organic (due to lesions to neuromuscular structures) or functional (associated with relaxation of the muscular apparatus) ${ }^{11}$. FNMES has been used for increasing muscle strength, decreasing spasticity, and controlling movement of limbs for many years. Numerous studies have suggested that neuromuscular stimulation reduces spasticity and enhances the muscle strength of the hemi paretic limb. ${ }^{12-}$

${ }^{13}$.A recent meta-analysis of four randomized trials concluded that neuromuscular stimulation improves the motor strength of stroke survivors. ${ }^{13}$

FNMES has been developed for restoring function in the upper extremity, lower extremity, respiratory system, bladder and bowel. Approximately, half of all stroke survivors are left with major functional problems in the hand and arm. Electrical stimulation of the wrist in combination with other rehabilitation strategies can result in increased grip strength and improved motor function.

Neuromuscular stimulation can be broadly categorized as therapeutic or functional. Therapeutic neuro-muscular stimulation is administered to obtain repetitive stimulation of paralyzed muscle to minimize specific impairments such as limited range of motion, motor weakness, spasticity and cardio vascular deconditioning. Although, therapeutic neuro-muscular stimulation may, and hopefully will, lead to functional improvements, the electrical stimulation does not directly provide function. Functional neuromuscular stimulation (FNMS) is defined as the use of electrical stimulation to activate paralyzed muscles in a precise sequence to assist in the performance of activities of daily living. Devices or system that provides FNMS are also appropriately called neuroprostheses. ${ }^{10}$

The goal of therapeutic exercise for the muscle re education and facilitation is to re-establish voluntary control of body positions and movements after injury or disease has affected the motor control mechanism. Motor control may be affected by damage to either or both the afferent and efferent neural pathways, as well as damage to central control centers in the motor and premotor cortex. Although, exact mechanisms are not clear, the nervous system is continually adapting to environmental stimuli. This re-organization is termed as neural plasticity. The peripheral and central nervous system (CNS) are capable of remarkable recovery in response to injury, through the processes of collateral sprouting and synaptic reclamation. $^{13}$

There are several studies that have been done to improve motor function by using functional neuromuscular stimulation. Literature reveals that FNMES improves motor recovery in acute stroke survivals. But many of the studies were concentrating on the improvement of gait by giving FNMES to the lower limb muscles. By keeping this fact in view the present study has been considered so as to improve hand function in stroke patients by giving FNMES along with conventional physiotherapy

\section{Materials \& Methodology :}

This study was approved by the Institutional Ethical Committee of Nitte Institute of Physiotherapy. 30 subjects including male and female from the outpatient 
department of K S Hegde Hospital, Mangalore, who were diagnosed by the Neurologist as having stroke were selected for the study. Subjects were included if, Stroke survivors admitted to hospital within 4 weeks of their stroke, Unilateral hemiparesis, $45-75$ yrs of age during the study, Mild to moderate deficits defined by Orpington prognostic scale 2.0 to 5.2 and patients with Folstein mini mental score more than 14 . Written consent was taken before conducting the study. We excluded the subjects if they had potentially fatal cardiac arrhythmias, cardiac pace makers, seizures within 2 years before admission, active reflex sympathetic dystrophy, prior stroke with residual motor weakness, lower motor neuron lesion of the impaired upper extremity, spinal cord injury, traumatic brain injury, multiple sclerosis, parkinsonism, sensory deficits, peripheral nerve injury. Subjects were randomly assigned after fulfilling inclusion criteria to either group 1 or 2 where; Group 1 patients ( Control) received only conventional Physical therapy for 1 hour per day for 6 days in a week for a period of 4 weeks and the Group 2 patients ( Experimental) received an additional functional neuromuscular Electrical stimulation along with conventional Physical therapy for 1 hour per day for 6 days in a week for a period of 4 weeks, which comprises of 20 minutes of FNMES and 40 minutes of conventional physical therapy. ${ }^{14}$ Both the groups were having 15 patients each.

All the patients were assessed before administering the intervention and at the end four weeks of intervention with the outcome tools as Box and Block Test (BBT) and Action Research Arm Test (ARAT) to know the improvements. Both the outcome scales were used to assess the hand function. The routine physiotherapy interventions were given to both the groups like; passive movements, active assisted movements, stretching and strengthening exercises. As patient's ability improved they were made to do activities of self care.

Before starting the FNMES treatment, the nature of treatment and sensation (tingling sensation leading to muscle contraction) was explained and a written consent was taken from them. ${ }^{15,16}$ The duration of treatment as well as any particular cooperation required was indicated. A 2-
Channel FNMES was used for the treatment where, the Channel 1was placed on extensor aspect i.e. the reference electrode and active electrode was placed on the proximal and distal end of the muscle bulk and the Channel 2 was placed on flexor aspect, reference electrode and active electrode was placed on the proximal and distal part of the muscle bulk. Machine was programmed on cyclic stimulation ${ }^{17-20}$. Patients were seated on a chair with arms raised and supported on the couch with his/her forearm supported in an initial position of wrist flexion. Patients received stimulation for $\mathbf{2 0}$ minutes and gradually time was decreased. Patients received stimulation to both flexor and extensor group of muscles alternately. The machine was programmed to give $20 \mathrm{sec}$ each of flexor and extensor muscle stimulation. Patients were asked to do movements along with stimulation such as grasping and releasing of blocks of varied sizes, grasping a glass and sponge of varied size.

The Action Research Arm Test (ARAT) was used to measure upper extremity function. The test was designed for use with people following stroke .The test comprises of 4subscales (grasp, grip, pinch and gross movement) each of 19 tests is scored on 4-point ordinal scale. The total possible score is 57 where; 0 -Can perform no part of test, 1 - Performs test partially, 2 - Completes the test but takes an abnormally long time or has great difficulty and 3 Performs the test normally ${ }^{21-24}$. In the BBT, a box with partition was taken with wooden blocks in it. In this the patients were given 15 seconds trial and then testing period of 60 seconds to move blocks from one side of the partition to other. Patients were seated such that his/her forearm was supported and box and block unit kept at the same level ${ }^{19,20}$. Patient picks up a block and releases on the other side by just crossing the partition. A time limit of 60 seconds are given to the patient. The blocks are counted at the end of the session.

\section{Results:}

The data were analyzed by using Wilcoxon signed rank sum test for the intra group comparison and Mann-Whitney $U$ test for the inter group comparison to know the improvement in hand function of Control and Experimental groups. 
Table 1: Intra group comparison of Action Research Arm Test for Control group

\begin{tabular}{|l|l|l|l|l|l|}
\hline & & $\mathrm{N}$ & $\mathrm{MEAN}$ & $\mathrm{S} \mathrm{D}$ & $\mathrm{Z}$ \\
\hline \multirow{2}{*}{$\begin{array}{l}\text { Group 1 } \\
\text { (Control) }\end{array}$} & PRE & \multirow{2}{*}{15} & 13.2000 & 11.4405 & -2.267 \\
\cline { 2 - 5 } \cline { 4 - 6 } & & 21.200 & 10.3593 & $\mathrm{p}=0.023$ \\
\hline
\end{tabular}

The Wilcoxon signed value is $-2.267(p=0.023)$ which was found to be statistically significant. Therefore, it is inferred that patients in the control group showed improvement in the hand function at the end of four weeks of conventional physical therapy when it was assessed on ARAT.

Table 2: Intra group comparison of Action Research Arm Test for Experimental Group.

\begin{tabular}{|l|l|c|l|l|l|}
\hline & & $\mathrm{N}$ & MEAN & $\mathrm{S} \mathrm{D}$ & $\mathrm{Z}$ \\
\hline $\begin{array}{l}\text { Group 1 } \\
\text { (Experimental) }\end{array}$ & PRE & \multirow{2}{*}{15} & 13.4000 & 12.0996 & -3.413 \\
\cline { 5 - 6 } \cline { 5 - 6 } & & & 39.8000 & 14.1835 & $\mathrm{p}=0.001$ \\
\hline
\end{tabular}

The Wilcoxon signed value is $-3.413(p=0.001)$ which was found to be statistically significant. Therefore, it is inferred that patients in the experimental group showed improvement in the hand function at the end of four weeks of conventional physiotherapy and an added FNMES when it was assessed on ARAT.

Table 3: Inter group comparison of Action Research Arm Test for Control and Experimental groups at the end of 4 weeks.

\begin{tabular}{|l|l|l|l|l|}
\hline Group & $\mathrm{N}$ & MEAN & SD & $\mathrm{U}$ value \\
\hline CONTROL & 15 & 10.2000 & 15.4401 & 33.0000 \\
\hline EXPERIMENTAL & 15 & 20.8000 & 0.5085 & $\mathrm{p}=0.001$ \\
\hline
\end{tabular}

The Mann-Whitney $U$ value was 33.0000 ( $p=0.001)$ which was found to be statistically highly significant. Therefore, it is inferred that the experimental group who received an additional FNMES showed significant improvement in the hand function when it was assessed on ARAT.

Table 4: Intra group comparison of Box and Block Test for Control group

\begin{tabular}{|l|l|c|l|l|l|}
\hline Group 1 & & $\mathrm{N}$ & MEAN & $\mathrm{S} \mathrm{D}$ & $\mathrm{Z}$ \\
\hline \multirow{2}{*}{ CONTROL } & PRE & \multirow{2}{*}{15} & 7.2000 & 6.30419 & 3.412 \\
\cline { 2 - 2 } \cline { 5 - 6 } & POST & & 21.6667 & 10.62791 & $\mathrm{p}=0.001$ \\
\hline
\end{tabular}

The Wilcoxon signed value is $3.412(p=0.001)$ which was found to be statistically significant. Therefore, it is inferred that patients in the control group showed improvement in the hand function at the end of four weeks of conventional physical therapy when it was assessed on BBT.

Table 5: Intra group comparison of Box and Block Test for Experimental group.

\begin{tabular}{|l|l|c|l|l|l|}
\hline Group & & $\mathrm{N}$ & MEAN & $\mathrm{S} \mathrm{D}$ & $\mathrm{Z}$ \\
\hline \multirow{2}{*}{ EXPERIMENTAL } & PRE & \multirow{2}{*}{15} & 8.3333 & 6.64042 & 3.412 \\
\cline { 2 - 2 } \cline { 5 - 6 } & POST & & 30.9333 & 12.64610 & $\mathrm{p}=0.001$ \\
\hline
\end{tabular}

The Wilcoxon signed value is $3.412(p=0.001)$ which was found to be statistically significant. Therefore, it is inferred that patients in the experimental group showed improvement in the hand function at the end of four weeks of conventional physical therapy along with FNMES when it was assessed on BBT.

Table 6 : Inter group comparison of Box and Block Test for Control and Experimental group at the end of four weeks.

\begin{tabular}{|l|l|l|l|l|l|}
\hline & GROUP & N & MEAN & SD & U Value \\
\hline POST & Control & 15 & 21.666 & 10.6279 & 2.0360 \\
\hline & Experimental & 15 & 30.933 & 12.6461 & $\mathrm{p}=0.041$ \\
\hline
\end{tabular}

The Mann-Whitney U value was 2.0360 ( $p=0.041$ ) which was found to be statistically highly significant. Therefore, it is inferred that the experimental group who received an additional FNMES showed significant improvement in the hand function when it was assessed on BBT.

\section{Discussion:}

This study was designed to investigate and compare the effectiveness of conventional physical therapy and conventional physical therapy along with functional neuromuscular electrical stimulation (FNMES) for improving the hand function in acute stage of stroke. The results of the study shows improvement in hand function in both the groups, but the, experimental group who received an additional FNMES along with conventional therapy showed a better improvement in hand function on the basis of ARAT scores and BBT scores.

Many researchers have stated that conventional physiotherapy which comprises of passive movements, active-assisted exercises, strengthening and stretching produces beneficial effects ${ }^{9}$. In Experimental group, the electrical stimulation was applied to the distal muscles that 
controlled the opening, grasping holding and releasing of objects $^{25}$. It was observed that subjects of the experimental group picked more blocks than control group when tested with BBT. Many researchers stated that active neuromuscular stimulation is an effective behavioral intervention for recovery after stroke and patients recovered better with FNMES than without it for lower limb muscles for improving gait patten. ${ }^{26-28}$

Nearly all studies on the recovery of motor function in stroke survivors have found that the most rapid recovery occurs during the first few weeks after stroke ${ }^{29}$.In a metaanalysis of 36 clinical trials in stroke rehabilitation, Ottenbacher and Jannell ${ }^{30}$ noted that early initiation of rehabilitation for stroke patients was related to improved motor and functional outcomes. These results suggested that early and intensive intervention could significantly improve motor recovery and functional outcome in stroke survivors. In the present study, FNMES was applied within four weeks after the stroke. There was no significant difference in subjects' characteristics before treatment. Thus, any differences among the 2 groups could be largely attributed to the effects of intervention.

In our study, FNMES was delivered reciprocally to the both groups of forearm muscles to mimic normal hand function. The possible mechanisms for the effects of FNMES in subjects with Stroke could be, the increase of synaptic efficacy in existing neural circuits, or formation of new synapses, may be involved in the earlier stages of motor learning according to Asanuma and Pavlides ${ }^{31}$. In addition, frequently repeated movements of the affected upper extremity of stroke subjects, induced by FNMES in this study, might reinforce network connection patterns. As Classen et $a^{32}$ noted, the phenomenon of motor cortical rearrangements could be the first step in skill acquisition. Such brain plasticity could underline improvements seen in the FNMES group.

Nudo et $\mathrm{al}^{33}$ have suggested that afferent input associated with repetitive movements facilitates improvement of motor function. For this reason, motor stimulation might be more effective in improving motor control than sensory stimulation would be. This increased effectiveness is likely due to electrical stimulation that provokes motor activation being associated with cutaneous, muscle and joint proprioceptive afferent feedback. In another way, the mechanism underlying power assisted FNMES therapy is that alternative motor pathways are recruited and activated to assist impaired afferent pathways. This explanation is based on the sensory motor integration theory that sensory input from movement of an affected limb directly influences subsequent motor output. As patients voluntarily attempt to extend the affected wrist and fingers, FNMES induces movement, and full extension is obtained. The results shows that FNMES in combination with conventional therapy may enhance the benefits of standard neurorehabilitative treatments and may also facilitate motor learning. Patients receiving motor, proprioceptive and cognitive inputs through the daily use of FNMES may demonstrate significantly greater improvements in voluntary movement and functional use of the hand.

The study was designed for acute stroke survivals and thus there were long term benefits out of it, as patient does not develop any contractures or deformities because the intervention was started soon after the onset of stroke and the subject himself was encouraged to generate muscle tension along with functional electrical stimulation.

Generalization of the results from this study should be performed with caution because of subject selection criteria, which did not cover all stroke categories or subjects aged younger than 45 or older than 75 years because it has been reported that stroke survivors with lower sensorimotor function have a decreased potential for recovery than do patients who are less severely affected.

Furthermore, more significant differences might have been detected earlier if the sample size were larger. To conclude, four weeks of FNMES, given 20 minutes per session with conventional physiotherapy, 6 days per week, improved the hand function in acute stroke subjects, more than conventional physiotherapy alone. 


\section{Conclusion :}

Authors concluded that at the end of 4 weeks of treatment when control and experimental groups were compared for improvement in hand function in acute stroke survivals; it was observed that experimental group who received an additional FNMES along with Conventional Physical therapy has showed significant improvement than control group. Therefore, adding an FNMES along with the

\section{References:}

1. K. Park: Preventive and Social Medicine, $16^{\text {th }}$ Edition, 2000, Pg no 208 283. Jabalpur, Banarasidas Bhanot

2. PK Sethi, "Management of Ischemic stroke". Neurosciences Today 2002; 6:139-143.

3. K. Anand, D. Chowdhury, K.B. Singh, C.S. Pandav, S.K. Kapoor, Estimation of Mortality and Morbidity due to Strokes in India. Neuroepidemiology 2001;20:208-211 (DOI: 10.1159/000054789)

4. Patricia A. Downie, Cash's textbook of Neurology for Physiotherapists, $4^{\text {th }}$ edition,1993, Page 241.

5. Paul Taylor, Jane Burridge, Sean Hagon, Paul Chappel, Ian Swain Improvement in hand function and sensation in chronic stroke patients following electrical stimulation exercises- A pilot study. www.salisburyfes.com/hand2.htm

6. Fatima De N A, P.Shelton, Michel J Reading. Effect of lesion location on upper limb motor recovery after stroke. Stroke, 2001 Jan: 32(1):107-12.

7. James Cauraugh, PhD; Kathye Light, PhD, PT; Sangbum Kim, MS; Mary Thigpen, PT, MHS Andrea Behrman, PhD, PT , Chronic Motor Dysfunction After Stroke. Recovering Wrist and Finger Extension by Electromyography-Triggered Neuromuscular Stimulation. Stroke. 2000;31:1360-1364

8. Yan T, Hui- Chan CW, Li Ls. Functional electrical stimulation improves motor recovery of the lower extremity and walking ability of subjects with first acute stroke: a randomized placebo-controlled trail. Stroke 2005 Jan: 36(1):80-5.

9. John Chae, MD; Francois Bethoux, MD; Theresa Bohinc, OTR; Loreen Dobos, PT; Tina Davis, OTR; Amy Friedl, OTR, Neuromuscular Stimulation for Upper Extremity Motor and Functional Recovery in Acute Hemiplegia. Stroke 1998;29;975-979

10. Merletti R, Zelaschi F, Latella D, Galli M, Angeli S, Sessa MB. A contro study of muscle force recovery in hemiparetic patients during treatment with functional electrical stimulation. Scand J Rehabil Med. 1978; 10:147-154.

11. Bowman BR, Baker LL, Waters RL. Positional feedback and electrica stimulation: an automated treatment for the hemiplegic wrist. Arch Phys Med Rehabil. 1979; 60:497-502.

12. Winchester P, Montgomery J, Bowman B, Hislop H. Effects of feedback stimulation training and cyclical electrical stimulation on knee extension in hemiparetic patients. Phys Ther. 1983; 63:1096-1103.

13. Kraft GH, Fitts SS, Hammond MC. Techniques to improve function of the arm and hand in chronic hemiplegia. Arch Phys Med Rehabil. 1992; 73:220-227.

14. Dimitrijevic M, Soroker N. Mesh-glove, II: Modulation of residual upper limb motor control after stroke with whole-hand electric stimulation. Scand J Rehabil Med. 1994; 26:187-190.

15. Faghri P, Rodgers M, Glaser R, Bors J, Ho C, Akuthota P. The effects of functional electrical stimulation on shoulder subluxation, arm function recovery, and shoulder pain in hemiplegic stroke patients. Arch Phys Med Rehabil. 1994; 75:73-79.

16. Dimitrijevic M, Stokic S, Wawro A, Wun C. Modification of motor control of wrist extension by mesh-glove electrical afferent stimulation in stroke patients. Arch Phys Med Rehabil. 1996; $77: 252-258$. conventional therapy is more effective in improving hand function in acute stroke survivals.

\section{Acknowledgements:}

Authors would like to thank the Department of Neurology and Department of Medicine of K S Hegde Hospital and the participants for their support.

Conflict of Interest : Authors agree that there was no source of conflict of interest.

17. Glanz M, Klawansky S, Stason W, Berkey C, Chalmers T. Functional electro stimulation in post stroke rehabilitation: a meta-analysis of the randomized controlled trials. Arch Phys Med Rehabil. 1996; 77:549-553.

18. Pomerov VM, King L, Pollock A, Bailey-Hallon A, Langhorne P. Electro stimulation for promoting recovery of movement or functional ability after stroke. Cochrane Database Syst Rev. 2006 Apr 19;(2):CD003241.

19. Ching- Lin Hsieh, I-Ping Hsueh, Fu-M Mei Chiang - Inter rater reliability and validity of the action research arm test in stroke patients. Oxford Journal; Age and Ageing, Vol 27 no 2 :Pg 107-113.

20. Vander Lee JH, Beckerman H, Lankhorst GJ, Bouter L M - The responsiveness of the action research arm test and Fugyl - Meyer assessment scale in chronic stroke patients. Journal of Rehabilitation Medicine; Mar 2001; 33(3): page 110-113.

21. Desrosier J, Bravo G, Hebert R, Dutil E, Mercier L-Validation of box and block test as a measure of dexterity of elderly people: Reliability, Validity and norms studies. Arch Phys Med Rehabil. 1994 Jul;75(7):751-5.

22. Darcy A.Umphred, Neurological rehabilitation. $4^{\text {th }}$ edition, 2001,pg 747.

23. De Kroon J, Lizerman M, Chae J. Relation between stimulation characteristics and clinical outcome in studies using electrical stimulation to improve motor control of the upper extremity in stroke. Journal of Rehabil. Med.2005, March; 37(2)65-74.

24. Baker LL, Yeh C, Wilson D. Electrical stimulation of wrist and fingers for hemiplegic patients. Phys Ther, 1979 Dec: 59(12):1495-9.

25. Wany Ry,Yang YR,Tsai MW. Effects of functional electrical stimulation on upper limb motor function and shoulder range of motion in hemiplegic patients. AMJ Phys Med Rehabil 2002 Apr: 81 (4):283-90.

26. Duncan P, Studenski S, Richards L, Gollub S, Lai SM, Revev D, Perera S, Yates J, Koch V, Rigler S, Johnson D, "Randomized clinical trial of therapeutic exercise in sub-acute stroke. Stroke 2003 Sep; 34 (9): $2173-80$

27. Studenski SA, Wallace D, Duncan PW, Rymer M, Predicting stroke recovery: three- and six-month rates of patient-centered functional outcomes based on the Orpington Prognostic scale. ": J Am Geriatr Soc. 2001 March; 49(3):308-12.

28. Johanna H. van der Lee, Heleen Beckerman, Gustaaf J. Lankhorst, Lex M. Bouter; The responsiveness of the action research arm test and the fugylmeyer assessment scale in chronic stroke patients. Journal of Rehabilitation Medicine, Volume 33, Number 3 / July 1, 2001.Pg 110-113.

29. Tiebin Yan, Christina W. Y. Hui-Chan and Leonard S. W. Li: Functional Electrical Stimulation Improves Motor Recovery of the Lower Extremity and Walking Ability of Subjects With First Acute Stroke : A Randomized Placebo-Controlled Trial; Stroke. 2005;36:80-85

30. Ottenbacher $\mathrm{KJ}$, Jannell $\mathrm{S}$. The results of clinical trials in stroke rehabilitation research. Arch Neurol. 1993; 50:37-44.

31. Asanuma $H$, Pavildes C. Neurobiological basis of motor learning in mammals. Neuroreport. 1997;8:1-4.

32. Classen J, Liepert J, Wise SP, Hallett M, and Cohen LG. Rapid plasticity of human cortical movement representation induced by practice. J Neurophysiol. 1998;79:1117-1123.

33. Nudo RJ,Wise BM, SiFuentis F et al; Neural substrates for the effects of rehabilitative trainings on motor recovery after ischaemic infarct. J.Science 1996;272:1791-1794 